\title{
Quad Band Rectangular Microstrip Antenna for S and C-Band Applications
}

\author{
Ambresh P. A., A. A. Sujata, A. M. Khan, P. M. Hadalgi, and P. V. Hunagund
}

\begin{abstract}
This paper presents the study of two rhombus shape slot loaded rectangular microstrip antenna for quad band operation. The quad bands are achieved at $S$ and $C$ band of frequencies. Effect of slot embedded on the patch is studied experimentally for enhancing the bandwidth. It is found that by using two rhombus shape slots on the patch element with copper as ground plane, the bandwidth at $\mathrm{C}$-band is enhanced from $2.06 \%$ to $20.4 \%$ without much affecting the operating bandwidth at $\mathrm{C}$-band. Further enhancement of bandwidth at the C-band does not affect the nature of broadside radiation characteristics. Details of antenna design are described and experimental results are discussed. The proposed antennas may find applications for the systems operating at $\mathrm{S}$ and $\mathrm{C}$-bands.
\end{abstract}

Index Terms-Microstrip antenna, quad band, rhombus, VNA, bandwidth.

\section{INTRODUCTION}

The microstrip antennas (MSAs) are the most widely used for the last few years due to their attractive features such as light weight, low volume, ease in fabrication and low cost [1]. However, the major disadvantage associated with MSAs is their narrow bandwidth [1]-[2] which restricts their many useful applications. Numbers of studies have been reported in the literature for enhancing the bandwidth [3]-[6]. Further, the dual frequency patch antennas have gained wide attention in radar communication particularly in synthetic aperture radar (SAR), as they avoid the use of two separate antennas for transmit and receive applications. Variety of methods have been proposed to obtain dual band operation, such as by loading slits [7], using slots in the patch [8], loading the patch with shorting pins [9], using stacked patches [10] et al. But the antenna operating at more than two different bands of frequencies and their enhancement are found rare in the literature. Hence a simple patch with rhombus shape slot technique has been used in this study for constructing the proposed antennas useful for $\mathrm{S}$ and $\mathrm{C}$ band applications.

\section{DESCRIPTION OF ANTENNA GEOMETRY}

The art work of the proposed antennas are developed using computer software AutoCAD-2012 and are fabricated on low

Manuscript received January 20, 2014; revised April 25, 2014.

Ambresh P. A. and A. M. Khan are with the Dept. of Electronics, Mangalore University, Mangalore 574199, Karnataka, India (e-mail: ambreshpa@rediffmail.com).

A. A. Sujata is with the Dept. of Electronics, Godutai Engineering College for Women, Gulbarga. Karnataka, India.

P. M. Hadalgi and P. V. Hunagund is with the Microwave Research Laboratory, Department of Applied Electronics, Gulbarga University, Gulbarga-585106, Karnataka, India. cost glass epoxy substrate material of thickness $h=0.16 \mathrm{~cm}$ and permittivity $\epsilon=4.4$. Fig. 1 shows the geometry of conventional rectangular microstrip antenna (CRMA) which is designed for the resonant frequency of $3.5 \mathrm{GHz}$, using the equations available in the literature [1]. The substrate area of the CRMA is $A=M \times N$. The antenna is fed by using microstripline feeding. This feeding has been selected because of its simplicity and it can be simultaneously fabricated along with the antenna element. Fig. 1 consists of a radiating patch of length and width $(L \times W)$ of the patch are $(18.99 \times 26.92)$, quarter wave transformer of length $L_{t}$ and width $W_{t}$ used between the patch and $50 \Omega$ microstripline feed of length $L_{f}$ and width $W_{f}$. At the tip of microstripline feed, a $50 \Omega$ coaxial SMA connector is used for feeding the microwave power.

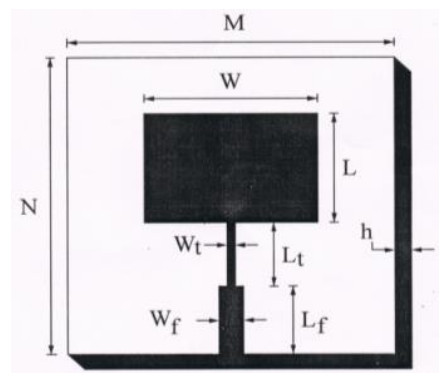

Fig. 1. Geometry of CRMA.

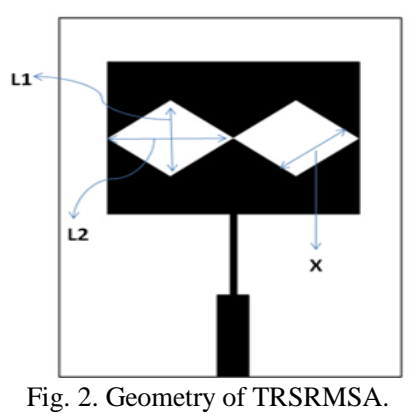

Fig. 2 shows the geometry of two rhombus shape slot rectangular antenna (TRSRMSA). The dimension of TRSRMSA shown in Fig. 2 remains same as that of rectangular patch and feed line as shown in Fig. 1, but two rhombus shaped slot which are placed horizontal on patch are etched on the patch plane of CRMA as shown in Fig. 2. Hence, this antenna is named as two rhombus shape slot rectangular antenna (TRSRMSA). The dimensions of all the slots are taken in terms of $\lambda_{0}$, where $\lambda_{0}$ is the free space wavelength corresponding to the designed frequency of conventional RMA i.e. $3.5 \mathrm{GHz}$. The length and width $(L \times W)$ of the patch are $(18.99 \times 26.92)$. The side length $x$ is $6.8 \mathrm{~mm}$. The horizontal and vertical slot lengths $\left(L_{1}\right.$ and $\left.L_{2}\right)$ slots are 9.6 $\mathrm{mm}$ and $13.5 \mathrm{~mm}$. 


\section{EXPERIMENTAL RESULTS}

The S-parameters of passive and active networks can be measured with a vector network analyzer (VNA), which is a two (or four) channel microwave receiver designed to process the magnitude and phase of the transmitted and reflected waves from the network. It compares the incident signal that leaves the analyzer with either the signal that is transmitted through the test device or the signal that is reflected from its input. The results of return loss, VSWR and impedance presented in this thesis are taken on VNA (Rohde \& Schwarz, Germany make, ZVK model 1127.8651, 10 MHz-40 GHZ).

The impedance bandwidth over return loss less than $-10 \mathrm{~dB}$ for the proposed antennas is measured at $\mathrm{S}$ and $\mathrm{C}$ band of microwave frequencies. The variation of return loss versus frequency of CRMA is as shown in Fig. 3. From the figure it is clear that, the antenna resonates at $f_{r 1}=3.6 \mathrm{GHz}$ of frequency which is very much close to the designed frequency of 3.5 $\mathrm{GHz}$ and hence validates the design. From this graph, the experimental impedance bandwidth is calculated using the formula,

$$
\mathrm{BW}=\left[\frac{f_{H}-f_{L}}{f_{C}}\right] \times 100 \%
$$

where, $f_{H}$ and $f_{L}$ are the upper and lower cut-off frequency of the band respectively when its return loss becomes $-10 \mathrm{~dB}$ and $f_{c}$ is the center frequency between $f_{H}$ and $f_{L}$. Hence by using (1) the bandwidth $\mathrm{BW}_{1}$ of CRMA is found to be $2.06 \%$. The theoretical bandwidth of this antenna is calculated using [2],

$$
\text { Bandwidth }(\%)=\left[\frac{A \times h}{\lambda_{0} \sqrt{\varepsilon_{r}}}\right] \times \sqrt{\frac{W}{L}}
$$

where, $A$ is the correction factor, which is found to be 180 as per [11]. The theoretical bandwidth of CRMA is found to be $3.42 \%$, which is in good agreement with the experimental value. Fig. 4 shows the variation of return loss versus frequency of TRSRMSA. The antenna resonates for four band of frequencies, $f r_{1}=2.63 \mathrm{GHz}, f r_{2}=4.64 \mathrm{GHz}, f r_{3}=5.94 \mathrm{GHz}$ and $f r_{4}=7.78 \mathrm{GHz}$. The respective bandwidths at $f r_{1}, f r_{2}, f r_{3}$ and $f r_{4}$ are $1.52 \%, 1.07 \%, 2.02 \%$ and $20.4 \%$. It is clear that the $\mathrm{BW}_{1}$ lies in the $\mathrm{S}$-band $(2-4 \mathrm{GHz})$, where as $\mathrm{BW}_{2}, \mathrm{BW}_{3}$ and $\mathrm{BW}_{4}$ lies in the $\mathrm{C}$-band $(4-8 \mathrm{GHz})$.

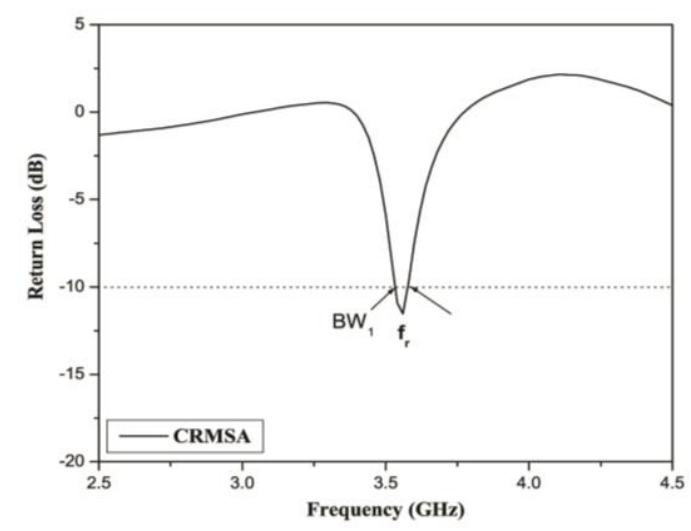

Fig. 3. Variation of return loss Vs frequency of conventional RMSA.

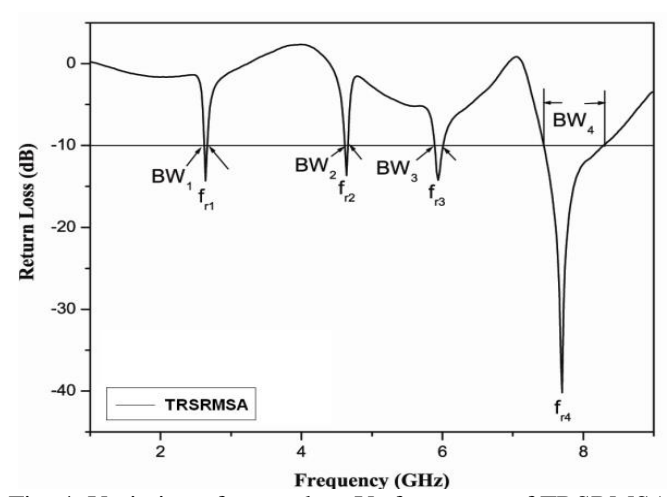

Fig. 4. Variation of return loss Vs frequency of TRSRMSA

Hence the construction of TRSRMSA does not affect the basic resonant property of antenna that is the primary band $\mathrm{BW}_{1}$ which lies at $\mathrm{S}$-band but gives other three bands $\mathrm{BW}_{2}$, $\mathrm{BW}_{3}$ and $\mathrm{BW}_{4}$ at $\mathrm{C}$-band. However, it is seen that the resonant frequency $f r_{2}$ of TRSRMSA in the primary band shifts to 4.64 $\mathrm{GHz}$, when compared to resonant frequency $f r_{1}$ of CRMA i.e. $2.63 \mathrm{GHz}$ in $\mathrm{BW}_{1}$. The shift of resonant frequency is mainly due to feed used in TRSRMSA. The dual bands are due to independent resonance of patch and slot elements in TRSRMSA. Hence it is clear that, TRSRMSA is quite effective in enhancing the bandwidth of antenna at S, C-band retaining the resonant property of antenna.

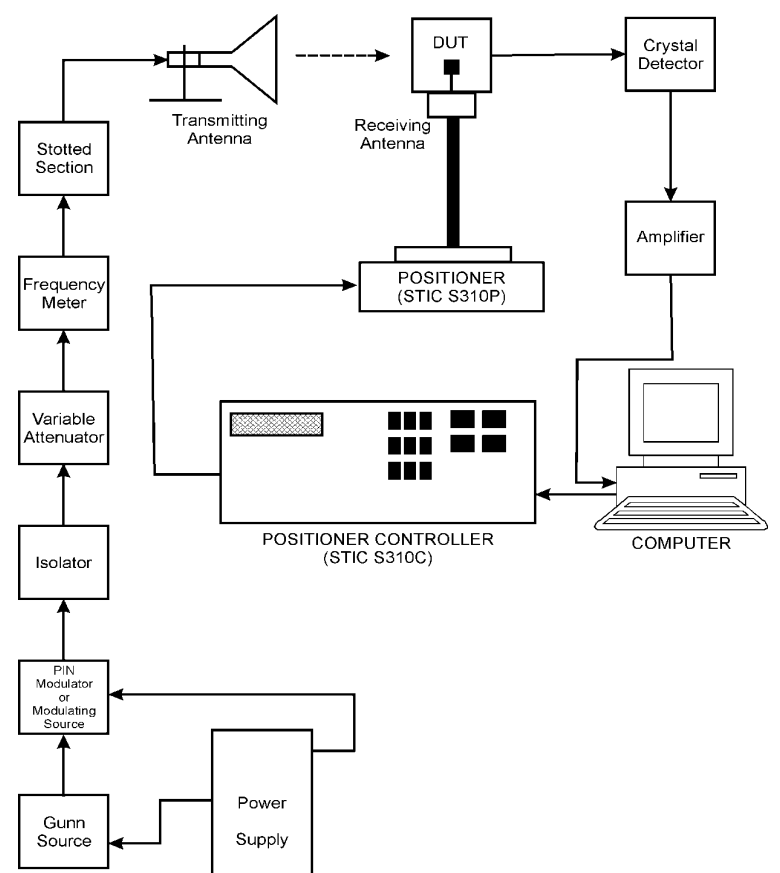

Fig. 5. PC based radiation pattern measurement setup.

The radiation patterns for CRMA and TRSRMSA are measured using basic microwave measurement setup and position control system (S310C) and antenna positioner (S310P) as shown in Fig. 5. Fig. 6 and Fig. 7 show the co-polar and cross-polar radiation patterns of CRMA and TRSRMSA, which are measured at their respective resonant bands. From these figures, it is clear that the patterns are broadsided and linearly polarized. The quad band operation and enhancement of bandwidth does not affect the nature of broadside radiation characteristics. For the calculation of gain of antenna under test (AUT), the power transmitted ' $P$ ' by pyramidal horn antenna and power received ' $P_{r}$ ' by AUT are 
measured independently [11]. With the help of these experimental data, the maximum gain $G(\mathrm{~dB})$ of CRMA in $\mathrm{BW}_{1}$ is calculated using (3).

$$
(G) d B=10 \log \left(\frac{P_{r}}{P_{t}}\right)-\left(G_{t}\right) d B-20 \log \left(\frac{\lambda_{0}}{4 \pi R}\right) d B
$$



Fig. 6. Measured radiation pattern of CRMA measured at $3.8 \mathrm{GHz}$.

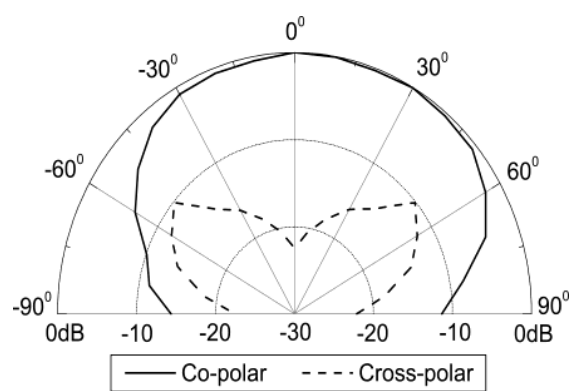

(a) $f r_{1}=2.63 \mathrm{GHz}$

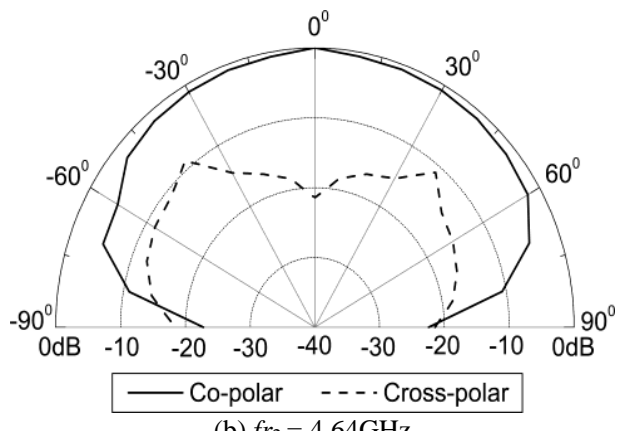

(b) $f r_{2}=4.64 \mathrm{GHz}$

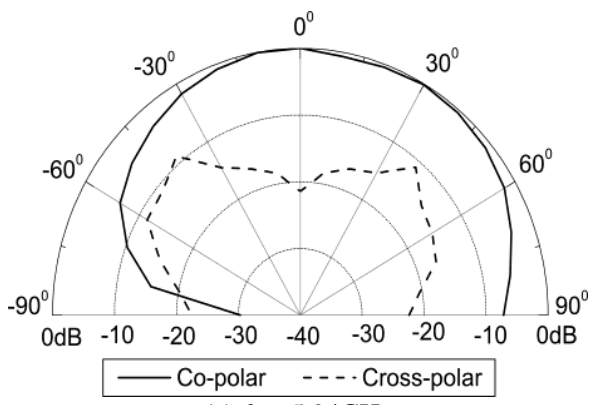

(c) $f r_{3}=5.94 \mathrm{GHz}$

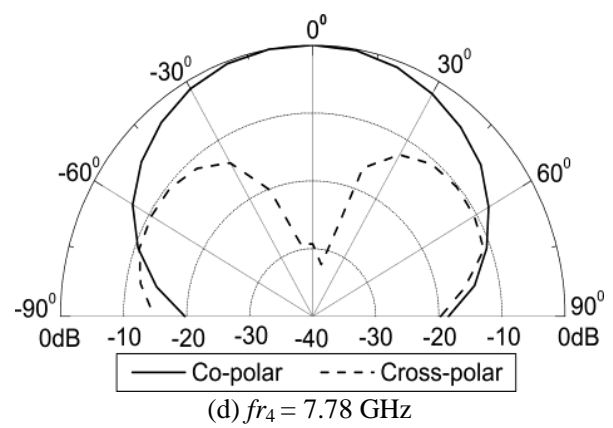

Fig. 7. Measured radiation patterns of TRSRMSA. where, $\lambda_{0}$ is the operating wavelength in $\mathrm{cm}$ and $R$ is the distance between the transmitting and receiving antenna. The gain of CRMA is found to be $2.3 \mathrm{~dB}$. The gains of TRSRMSA is measured for all resonant frequencies and are found to be $2.6 \mathrm{~dB}, 3.1 \mathrm{~dB}, 3.8 \mathrm{~dB}$ and $6.3 \mathrm{~dB}$ respectively. The maximum gain is found to be $6.3 \mathrm{~dB}$ for $7.78 \mathrm{GHz}$. The proposed TRSRMSA finds application in S and C frequency band of wireless communication.

The logical variables used at the microwave frequency are traveling waves rather than total voltage and total currents. The basic task of vector network analyzer (VNA) is the measurement of S-parameters. The photography of VNA is as shown in Fig. 8.



Fig. 8. The photography of vector network analyzer (VNA).

\section{CONCLUSION}

This paper presents the study, design of quad band operation of antenna at four different bands of frequencies which is possible by constructing two rhombus slots on rectangular microstrip patch element. Effect of rhombus slot of different shapes has been studied experimentally for enhancing the bandwidth. It is found that, the bandwidth at the C-band is enhanced to $20.4 \%$ without much affecting the primary band. The enhancement of bandwidth at S, C-band does not affect the nature of broadside radiation characteristics. The proposed antennas are simple in their design and construction and they use low cost substrate material. These antennas may find applications for the systems operating at $\mathrm{S}$ and $\mathrm{C}$-band of frequencies.

\section{ACKNOWLEDGMENT}

Authors would like to express gratitude to The Department of Science and Technology (DST), Government of India, New Delhi, for sanctioning Vector Network Analyzer to this Department under FIST Project

\section{REFERENCES}

[1] I. J. Bahl and P. Bhartia, Microstrip Antennas, Artech House, MA, 1981.

[2] D. M. Pozar, "Microstrip antennas," Proceedings of the IEEE, vol. 80, no. 1, p. 79, 1992.

[3] H. F. Pues and A. R. V. D. Capelle, "An impedance matching technique for increasing the bandwidth of microstrip antennas," IEEE Trans. on Antennas \& Propag, vol. 37, no. 11, pp. 1345- 1354, 1989.

[4] K. Oh, B. Kim, and J. Choi, "Design of dual and wideband aperture stacked patch antenna with double-sided notches," Electronic Letters, vol. 40, no. 11, pp. 643-645, 2004. 
[5] J. Y. Sze and K. L. Wong, "Slotted rectangular microstrip antenna for bandwidth enhancement," IEEE Trans. Antennas Propag, vol. 48, no. 8, pp. 1149-1152, 2000.

[6] G. Kumar and K. C. Gupta, "Broad-Band microstrip Antennas using additional resonators gap-coupled to the radiating edges," IEEE Trans. on Antennas and Propag., vol. 32, issue 12, pp. 1375-1379, 1984.

[7] Q. Q. Wang, B. Z. Wang, and J. He, "Wideband and dual- band design of a printed dipole antenna," IEEE Antennas Wireless Propag. Letters, vol. 7, pp. 1-4, 2008.

[8] Y. X. Guo, K. M. Luk, and K. F. Lee, "Dual band slot-loaded short-circuited patch antenna," Electron Letters (UK), vol. 36, no. 4, pp. 289-291, 2000.

[9] S. C. Pan and K. L. Wang, "Dual frequency triangular microstrip antenna with a shorting pin," IEEE Trans Antennas Propag., vol. 45, no. 12, pp. 1889-1891, 2002.

[10] K. Oh, B. Kim, and J. Choi, "Design of dual and wideband aperture stacked patch antenna with double-sided notches," Electron Letters (UK), vol. 40, no. 11, pp. 643-645, 2004.

[11] C. A. Balanis, Antenna Theory Analysis and Design, John Wiley and Sons, New York, 1982.



Ambresh P. A. received the M.Tech degree in communication systems engineering from Poojya Doddappa Appa College of Engineering, Gulbarga, Karanataka in the year of 2008. In April 2013, he also received Ph.D degree in the field of microwave electronics in the Department of P. G. Studies \& Research in Applied Electronics, Gulbarga University, Gulbarga, Karnataka. Currently, he is working as an Asst. Prof. in the Dept. of Electronics, Mangalore University, Mangalore. India. His research interest involves design, development and parametric performance study of microstrip antenna for RF/Microwave front-ends. He has published more than 40 papers in referred journals and conference proceedings. He is also researching antenna design for GPS/IMT-2000/WLAN/WiMax application.
A. A. Sujata received M.Tech degree in communication systems engineering from Poojya Doddappa Appa College of Engineering, Gulbarga, Karnataka. Since 7 years ago, she is working as an Asst. Prof in the Dept. of Electronics, Godutai Engineering College for Women, Gulbarga. Her research interests are communication systems, image processing. She has published 6 papers in referred journals and conference proceedings.

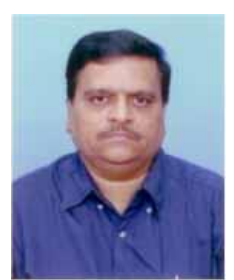

P. V. Hunagund received his M.Sc in Department of Applied Electronics, Gulbarga University, Gulbarga in 1981. In the year of 1992, he received Ph.D degree from Gulbarga University, Gulbarga. From 1984 to 1993, he was a lecturer in the Department of Applied Electronics, Gulbarga University, Gulbarga. From 1993 to 2003, he was a reader in Dept. of Applied Electronics, Gulbarga University, Gulbarga. In 2003 he got promoted under CAS as a professor. He has served as chairman of the department three times successfully. $6 \mathrm{Ph} . \mathrm{D}$ students have got their Ph.D degree under his guidance and 6 more are still working on it. He had published more than 200 papers in referred journals and conference proceedings.

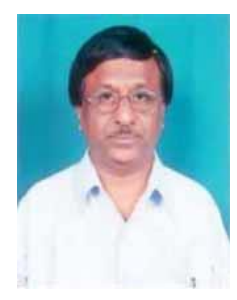

P. M. Hadalgi received the M.Sc and Ph.D degrees in the Department of P. G. Studies \& Research in Applied Electronics, Gulbarga University, Gulbarga in the year 1981 and 2006 respectively. From 1985 to 2001, he was a lecturer in the Department of Applied Electronics, Gulbarga University, Gulbarga. From 2001 to 2006, he was a Sr. Sc. lecturer in Dept. of Applied Electronics Gulbarga University, Gulbarga Recently he has been promoted as associate professor and chairman in the Department of Applied Electronics, Gulbarga University, Gulbarga. He had published more than 150 papers in referred journals and conference proceedings. His main research interest includes study, design and implementation of microwave antennas and front-end systems for UWB, WiMax, RADAR and mobile telecommunication systems. 